\title{
A new neovalve type in short bowel syndrome surgery
}

\author{
M. Zurita, J. M. Raurich ${ }^{1}$, A. Ramírez ${ }^{2}$ J. Gil ${ }^{2}$ and J. Darder ${ }^{3}$ \\ Department of General and Gastrointestinal Surgery. ${ }^{1}$ Unit of Intensive Care. ${ }^{2}$ Service of Microbiology. ${ }^{3}$ Service of \\ Anesthesiology. Hospital Universitario Son Dureta. Palma de Mallorca, Islas Baleares. Spain
}

\begin{abstract}
The objective of this study was to compare the clinical and analytical repercussion of a new type of intestinal valve -which can be used in both massive resections and right hemicolectomies requiring the elimination of the ileocecal valve- on two historical series of patients: one group with ileocolic resections and end-toend anastomoses (EE), and one group with Ricotta valves.

We compared 23 patients with ileocolic resection and end-toend anastomosis, 15 with Ricotta's valve, and 20 patients with a new valve made with a small intestinal invagination. There were no statistically significant differences in baseline characteristics among patients. Patients with the new valve showed less relevant weight loss and fewer stools at 3 months and one year when compared to patients with Ricotta's valve, and particularly those with ileocolic resection. Moreover, with the new valve technique there was neither colonization of the ileal mucosa nor bacterial overgrowth.

In conclusion, in massive intestinal resections and right hemicolectomies, including the ileocecal valve, the new valve technique would be of choice.
\end{abstract}

Key words: Short bowel syndrome. Ileocecal valve. Bacterial translocation.

Zurita M, Raurich JM, Ramírez A, Gil J, Darder J. A new neovalve type in short bowel syndrome surgery. Rev Esp Enferm Dig 2004; 96: 110-118.

\section{INTRODUCTION}

Short bowel syndrome (SBS) includes all pathophysiological symptoms and disorders triggered after massive intestinal resection (MIR), which may include all or a part of the colon. Most frequent causes include mesente-

Recibido: 24-06-03.

Aceptado: 02-10-03.

Correspondencia: Manuel Zurita y Romero. C/ San Roque, 9-3º izda. Tel.: 971715 293. 07001 Palma de Mallorca, Baleares ric infarction, Crohn's disease, regional enteritis, trauma, and tumors (1). The main clinical manifestations of SBS are diarrhea, weight loss, and malnutrition due to malabsorption, particularly when the preservation of the terminal ileum and ileocecal valve is rendered impossible.

Different surgical techniques have been applied to reduce the clinical manifestations of SBS, one of them being the formation of valves. With new valve surgical techniques, from a functional point of view we attempt to: a) reduce retrograde bacterial contamination (RBC); b) reduce bacterial overgrowth (BOG), and avoid possible bacterial translocation; c) decrease malabsorption and diarrhea; d) increase time of intestinal transit and absorption; e) reduce hyperoxaluria and the incidence of renal and bladder lithiasis; f) reduce weight loss; and g) provide an improved quality of life for the patient. Although clinical experience in the formation of new valves in SBS is limited, good results have been described (2-5).

\section{AIMS OF THE STUDY}

To compare the clinical and analytical repercussion of a new type of valve - which may be used in both MIR and right hemicolectomies requiring the elimination of the ileocecal valve- on two historical series of patients: one group with ileocolic resection and end-to-end anastomosis (EE), and the other with Ricotta's valve.

\section{MATERIAL AND METHODS}

\section{Description of the new valve technique by jejunal and/or ileocolic invagination}

The application of this new surgical technique to human pathology was carried out after experimental verification in adult dogs. After performing a medial laparo- 
tomy in patients, bowel areas to be resected were chosen and excised using an electric scalpel. Subsequently, 3-4 locking sutures were placed $1 \mathrm{~cm}$ away from the edge of the small intestine with a double-arm suture, and placed on the mesenteric, antimesenteric and side edges. These are passed 1 by 1 through the colon lumen and brought out in an anatomical situation similar to that corresponding to them in the small intestine. The small intestine invagination in the colon lumen $(8 \mathrm{~cm})$ is facilitated by the hand and the soft traction of the 3-4 locking sutures; subsequently, after checking there were no intestinal twists, the locking sutures were tied onto the serosa of the colon. We then carried out a circumferential crown of loose stitches with non-reabsorbable 3-0 material between the sectioned edge of the colon and the small intestine; once finished, we proceeded to take out the locking sutures, which allowed for an evagination of the invaginated small intestine in a slow but progressive way, which will virtually reach the colo-intestinal suture. Finally, we proceeded to close the meso-colic breach, placed a Penrosetype drainage, and closed the laparotomy (Fig. A, 1-5).

\section{Clinical studies}

Patients were divided into 3 groups according to surgical technique as applied in successive periods of time: a) ileocolic EE group: surgical procedure consisted of an ileo-transversotomy with EE anastomosis after intestinal resection, performed between 1975 and 1983; b) Ricotta group: application of a Ricotta's valve after intestinal resection, performed between 1984 and 1994; and c) new valve group: application of an ileal invagination in the colon lumen to form a new valve after intestinal resection, carried out throughout the period 1995-2000.

Ileocolic EE. This was performed in 23 patients with a mean age of 68 years (64-73), and a male-female ratio of 12:11. Twenty right colon cancers, 2 mesenteric ischemias, and 1 complicated Crohn's disease were operated on.

Ricotta. This was used in 15 patients with a mean age of 70 years (40-86), with a male-female ratio of 13:2. Two right colon cancers, 11 mesenteric ischemias, 1 complicated Crohn's disease, and 1 ileal lymphoma were operated on.

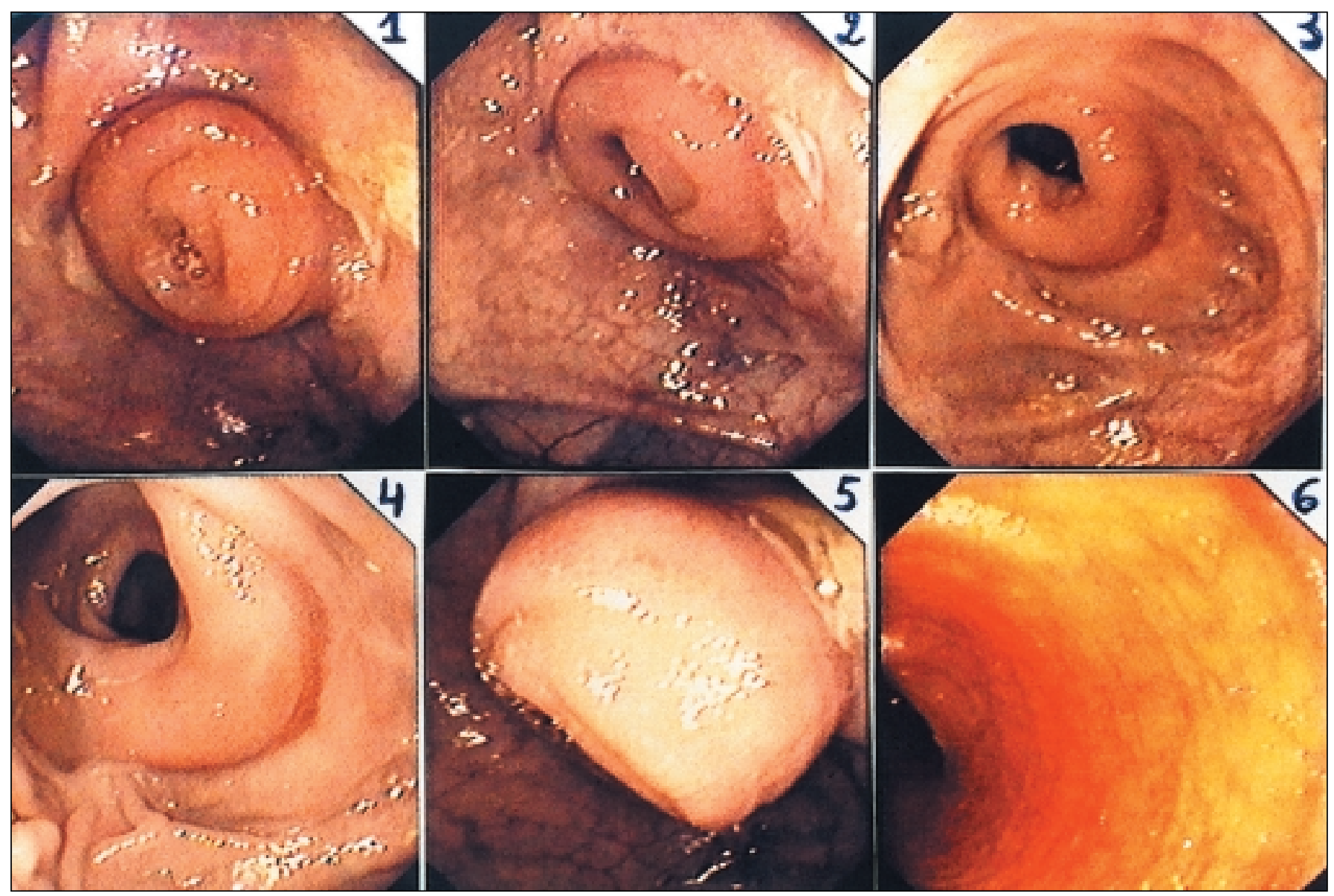

Fig. A.- 1: Placement of locking sutures. 2: lleocolic invagination and tying of locking sutures. 3: lleocolic anastomosis, closure of the mesocolic breach, and elimination of locking sutures. 4: lleocolic section: beginning the ileal mucosa evagination. 5: Cross section and view of the orifice in the ileal nipple. 6: Non-occlusive opaque enema and physiological reflux

1: Colocación de los puntos fiadores. 2: Invaginación ileocólica y anudación de los puntos fiadores. 3: Anastomosis ileocólica, cierre brecha mesocólica y eliminación puntos fiadores. 4: Sección ileocólica: inicio evaginación mucosa ileal. 5: Sección transversal y vista orificio en pezón ileal. 6: Enema opaco no oclusivo y reflujo fisiológico. 
New valve. This was carried out in 20 patients with a mean age of 70 years (59-83), with a male-female ratio of 12:8. It was performed for 15 right colon cancers, 3 mesenteric ischemias, 1 complicated Crohn's disease, and 1 ileal lymphoma.

Follow up, which was not possible for 3 patients ( 1 case of ileocolic EE anastomosis caused by mesenteric ischemia stopped attending the surgery; another case with a Ricotta's valve to treat a right-sided colon carcinoma rejected any type of exploration or study because of old age; a third case in which a new valve had been built up moved to a different Autonomous Community), consisted of opaque enema, colonoscopy, and a study of BOG using the hydrogen breath test with glucose at 3,6 and 12 months after surgery.

\section{Statistics}

Results are expressed as a mean \pm s.e.m. Statistical studies were carried out using the ANOVA test.

\section{RESULTS}

There were no statistically significant differences between-group regarding baseline weight (Table I). After 3 months there was some weight loss, which was lowest in patients in the group treated with the new valve technique, and highest in the group with the ileocolic EE technique (Table I). After 1 year, patients recovered weight, which was greatest in patients in whom the new valve technique was used and smallest in patients submitted to the ileocolic EE technique (Table I).

The number of postoperative stools after 3 months and
1 year was lowest with the new valve technique and highest with the ileocolic EE technique, and these differences reached statistical significance (Table I).

Serum bile acid (normal range: 0-8.1 $\mu \mathrm{mol} / \mathrm{l})$, ferritin (normal range: $30-400 \mathrm{ng} / \mathrm{dl}$ ) and zinc (normal range: 80 $130 \mu \mathrm{g} / \mathrm{dl}$ ) fell after 3 months, with a tendency to return to normal values at 1 year; this fall was most marked for the ileocolic EE technique. Serum copper (normal range: $70-140 \mu \mathrm{g} / \mathrm{dl}$ ) showed a rise in baseline value, which was greatest with the ileocolic EE technique (Table I).

When comparing patients who underwent a dilated right hemicolectomy to those who had MIR, the number of stools was lower with the new valve and Ricotta's techniques compared the ileocolic EE technique, and this lower intestinal rhythm was maintained over time (Table II). Between the new valve and the Ricotta's technique differences did not attain statistical significance (Table II).

Barium enemas performed in 17 patients with ileocolic EE anastomosis showed important retrograde filling in the small intestine. Fifteen patients in the new valve group and 10 in the Ricotta group had a continent valve, with neither obstruction nor reflux showing up in barium enema (Fig. A, 6).

Colonoscopies performed in 15 patients in the new valve group, 8 in the Ricotta group, and 9 in the ileocolic EE group showed that all patients with ileocolic EE anastomosis had colonization of the ileal mucosa; there was colonization of lesser intensity in 4 patients submitted to the Ricotta's technique, and absence of ileal colonization with the new valve technique (Fig. B, 1-5 and Fig. B, 6).

The results of BOG studies performed using the hydrogen breath test with glucose were positive in all patients at 3 months, and in 4 patients at 1 year, with the ileocolic EE technique; with the Ricotta's technique they were positive in 2 patients at 3 months, and in no patient

Table I. Comparison of clinical parameters by technique applied

\begin{tabular}{|c|c|c|c|c|}
\hline & Ileocolic E-E n: 23 & Ricotta n: 15 & Neo valve $n: 20$ & $p$ \\
\hline Initial weight & $67 \pm 6(59-80)$ & $67 \pm 7(58-81)$ & $70 \pm 4(62-78)$ & 0.10 \\
\hline Weight at 3 months & $51 \pm 5(42-66)$ & $57 \pm 6(50-69)$ & $64 \pm 4(57-73)$ & $<0.001$ \\
\hline Weight $\geq 1$ year & $58 \pm 5(52-68)$ & $63 \pm 7(51-76)$ & $68 \pm 4(61-76)$ & $<0.001$ \\
\hline Post-surgical stools & $4 \pm 1(3-5)$ & $3 \pm 0.7(2-4)$ & $2.4 \pm 0.5(2-3)$ & $<0.001$ \\
\hline Stools at 3 months & $3 \pm 0.3(2-3)$ & $2.3 \pm 0.4(2-3)$ & $1.8 \pm 0.4(1-3)$ & $<0.001$ \\
\hline Stools $\geq 1$ year & $2 \pm 0.4(1-3)$ & $1.5 \pm 0.5(1-2)$ & $1.2 \pm 0.4(1-2)$ & $<0.001$ \\
\hline Post-surgical ABTS $\mu \mathrm{mol} / \mathrm{l}$ & $1.2 \pm 0.6(0.2-2.3)$ & $1.5 \pm 0.5(1-2)$ & $2.5 \pm 0.9(0-4)$ & $<0.001$ \\
\hline ABTS at 3 months $\mu \mathrm{mol} / /$ & $0.9 \pm 0.5(0-1.7)$ & $1.6 \pm 0.6(0.9-2.8)$ & $1.3 \pm 0.4(0.6-2)$ & $<0.001$ \\
\hline ABTS $\geq 1$ year $\mu \mathrm{mol} / /$ & $1.5 \pm 0.6(0-3)$ & $2.6 \pm 0.7(1-3.6)$ & $2 \pm 0.5(1-3)$ & $<0.001$ \\
\hline Post-surgical ferritin $\mathrm{ng} / \mathrm{ml}$ & $206 \pm 94(78-365)$ & $277 \pm 110(59-375)$ & $239 \pm 111(59-375)$ & 0.57 \\
\hline Ferritin at 3 months ng/ml & $126 \pm 65(40-242)$ & $193 \pm 94(51-321)$ & $220 \pm 104(50-353)$ & 0.003 \\
\hline Ferritin $\geq 1$ year $\mathrm{ng} / \mathrm{ml}$ & $183 \pm 81(70-310)$ & $203 \pm 98(56-337)$ & $233 \pm 109(55-377)$ & 0.25 \\
\hline Post-surgical zinc $\mu \mathrm{g} / \mathrm{dl}$ & $96 \pm 13(78-123)$ & $101 \pm 16(83-137)$ & $101 \pm 15(80-130)$ & 0.35 \\
\hline Zinc at 3 months $\mu \mathrm{g} / \mathrm{dl}$ & $65 \pm 8(52-79)$ & $87 \pm 13(71-116)$ & $92 \pm 18(62-124)$ & $<0.001$ \\
\hline Zinc $\geq 1$ year $\mu \mathrm{g} / \mathrm{dl}$ & $81 \pm 10(69-100)$ & $92 \pm 14(74-122)$ & $103 \pm 14(79-127)$ & $<0.001$ \\
\hline Post-surgical copper $\mu \mathrm{g} / \mathrm{dl}$ & $105 \pm 17(77-141)$ & $110 \pm 16(79-130)$ & $104 \pm 15(77-130)$ & $<0.001$ \\
\hline Copper at 3 months $\mu \mathrm{g} / \mathrm{dl}$ & $152 \pm 35(100-243)$ & $126 \pm 17(91-150)$ & $112 \pm 16(83-140)$ & 0.51 \\
\hline Copper $\geq 1$ year $\mu \mathrm{g} / \mathrm{dl}$ & $110 \pm 14(72-142)$ & $118 \pm 16(86-140)$ & $104 \pm 15(72-128)$ & $<0.001$ \\
\hline
\end{tabular}

ABTS: total serum bile acid 
Table II. Clinical comparison between dilated R hemicolectomy and MIR-EE by surgical technique: new valve (NV), ileocolic endto-end resection (MIR-EE), and Ricotta's technique (Ricotta)

\begin{tabular}{|c|c|c|c|c|c|c|}
\hline & \multicolumn{3}{|c|}{$R$ hemicolectomy } & \multicolumn{3}{|c|}{$M I R$} \\
\hline & $N V$ & $M I R-E E$ & Ricotta's & $N V$ & MIR-EE & Ricotta's \\
\hline $\mathrm{N}^{\circ}$ patients & 13 & 20 & 2 & 7 & 3 & 13 \\
\hline $\mathrm{N}^{\circ}$ stools $<3$ months & $2.5^{\star}$ & 3.5 & 2.6 & $3 *$ & 6 & 3.5 \\
\hline $\mathrm{N}^{\circ}$ stools $>3$ months & $1.3 *$ & 2.5 & $1.5^{*}$ & $2 *$ & 5.5 & $2.2^{*}$ \\
\hline No stools ( $1-5$ years) & $1 *$ & 2.2 & 1 * & $1.2^{*}$ & 3.6 & $1.7 *$ \\
\hline Anastomotic spillage & 1 & 2 & 0 & 0 & 0 & 2 \\
\hline Death from operation & 0 & 0 & 0 & 0 & 0 & 0 \\
\hline Death from other reasons & 1 & 3 & 0 & $2++$ & 1 & 2 \\
\hline
\end{tabular}

${ }^{*} \mathrm{p}<0.05$ regarding MIR-TT.

++ patients with established multiple organ failure

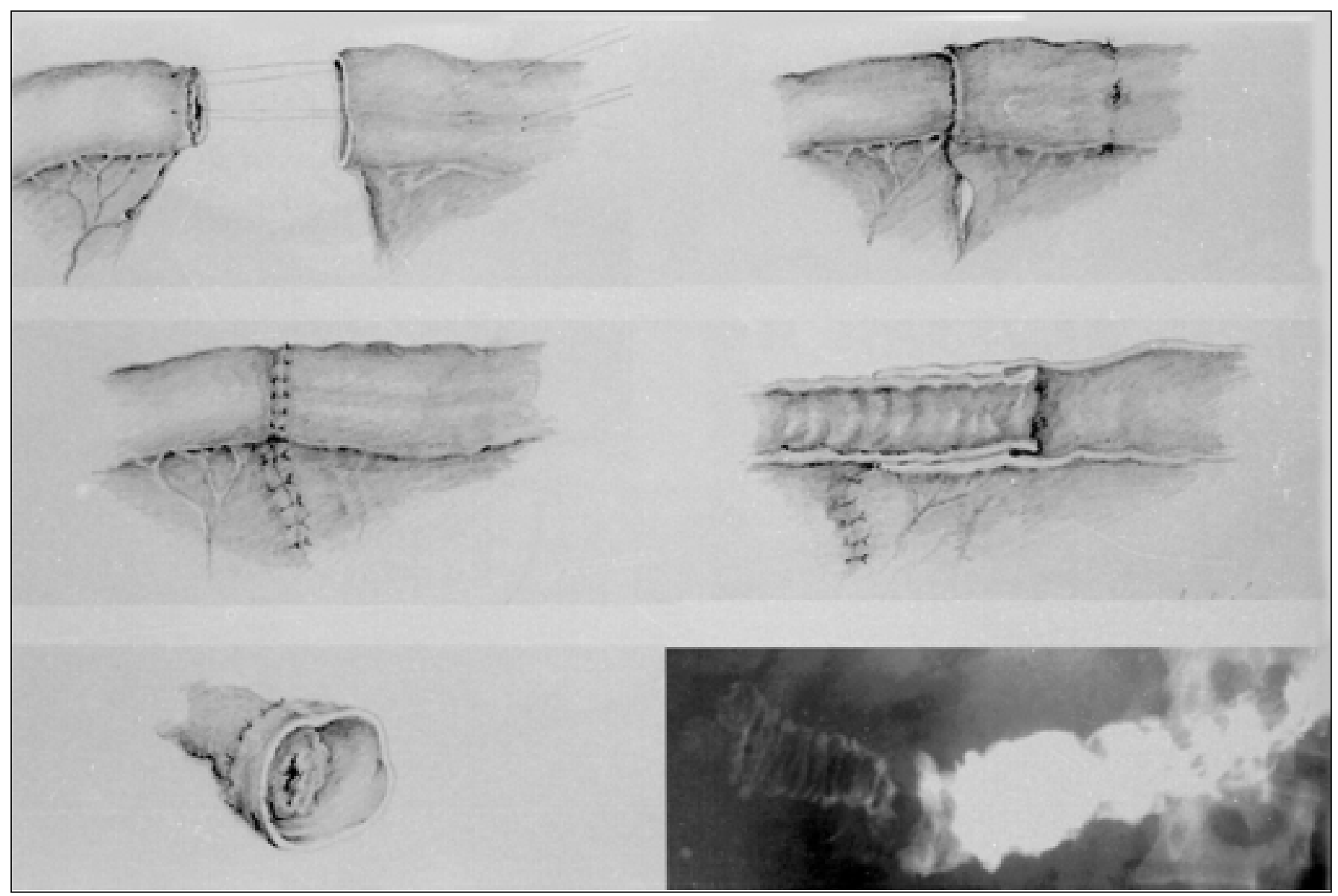

Fig. B.- 1: New valve at rest. 2: Beginning of the peristaltic valvular wave. 3: Partial opening of the valvular orifice. 4: Virtually total opening of the valvular orifice. 5: Maximum valvular expansion. 6: Passage of a colonoscope through the new valve, and a view of the normal ileal mucosa.

1: Neoválvula en reposo. 2: Inicio onda peristáltica valvular. 3: Apertura parcial orificio valvular. 4: Apertura casi total del orificio valvular. 5: Expansión máxima valvular. 6: Paso del colonoscopio a través de la neoválvula y visión de mucosa ileal normal.

at 1 year; with the new valve technique all patients were negative for BOG at 3 and 12 months.

\section{DISCUSSION}

The main results of the comparative study of these three surgical techniques indicate that patients develop lower weight loss, lower frequency of stools, and lower biochemical alterations with the new valve technique, as opposed to the ileocolic EE technique. Likewise, an absence of ileal mucosa colonization and bacterial retrograde contamination may be seen with the new valve technique, in contrast with the ileocolic EE technique.

Surgical techniques that have been used to reduce clinical symptoms in SBS include: a) antiperistaltic bowel 
Table III. Comparative parameters in clinical pathology and according to the technique applied

\begin{tabular}{|c|c|c|c|c|c|c|}
\hline & Ricotta's & Ribault & Vayre & Blanco & Casal & Authors \\
\hline Year & 1981 & 1990 & 1991 & 1994 & 1996 & 2000 \\
\hline Flora in lumen SI-LI & No & No & No & No & No & Yes \\
\hline Flora typical of intestinal mucosa & No & No & No & No & No & Yes \\
\hline Barium X-ray exam & Yes & No & Yes & Yes & Yes & Yes \\
\hline Intestinal transit time & Yes & Ns & Ns & Yes & Ns & Yes \\
\hline $\mathrm{N}^{\circ}$ stools/day & Yes & No & Yes & Yes & No & Yes \\
\hline Vesicular and renal echo & No & No & No & No & No & Yes \\
\hline Weigth & Yes & No & Yes & Yes & No & Yes \\
\hline Obstruction/fistula & Yes & Yes & No & No & No & No \\
\hline Retrograde colonization & Yes & Ns & Ns & Ns & No & No \\
\hline BOG: exhaled air test & No & No & No & No & No & Yes \\
\hline SteNotic syndrome & Yes & Yes & No & No & No & No \\
\hline Post-operative mortality & Ns & $15 \%$ & $18 \%$ & No & No & $15 \%$ \\
\hline
\end{tabular}

SI: small intestine; LI: large intestine; ns: not specified; BOG: bacterial overgrowth.

$(6-8)$; b) recirculation bowel $(9-11)$; c) retrograde pacemaker $(12)$; d) reduction of caliber and intestinal plication $(13,14)$; e) intestinal myotomy $(15,16)$; f) intestinal bags (17); g) perintestinal rings (18); h) intestinal neomucosa growth (19); i) small bowel transplantation $(20) ; \mathrm{j}$ ) reduction of caliber and small bowel enlargement $(21) ; \mathrm{k}$ ) sequential enlargament (22); and 1) valve formation.

In experimental studies, different types of valves have been used with better survival. The first bibliographic study found is that by Kellog (23) and, based on these works, Ricotta (24) in 1981 -after carrying out an emergency operation in a child with MIR- performed a nipple type jejunum-colic intubation, $8 \mathrm{~cm}$ in length, on 2 planes. Subsequently he performed an experimental study in dogs with a valve, and saw its braking effects. After experimental and clinical verifications, we may now point out that a reestablishment of intestinal transit was simple with hardly any morbidity or mortality, and this invagination may be compared to an ileal valve. When the ileal mucosa evaginates, it comes into contact with the colon mucosa until it reaches the level of the external coloileal suture line, thus avoiding the risk of stenosis by coalescence of the edges of the ileal invagination; it is the ileal mucosa that is in contact with the intracolic contents, which is preferable to the serous surface. This ileal evagination allows a restoration of transit after MIR, which makes it useful in SBS. We believe that this new valve is continent, and allows that all goals of the study be attained.

Historical review of the formation of some types of new valves (Table III): a) Kellog (23) tries to repair an incompetent ileocecal valve with an invagination of the terminal ileum in the ascending colon; b) Ricotta (24) sets up an ileocolic new valve in SBS for a child after experimental studies, and in clinical applications found a lower weight loss and the presence of BOG in the residual small intestine; c) Canarelli (2) forms a hemivalve following Ricotta's technique. Transit time is reduced with no occlusions found; d) Ribault (3) carries out a Ricotta's type ileocolic intubation on a colon mucosa hammock in complicated intestinal typhoid diseases. He reports few complications (3\% occlusion and fistula, and $15 \%$ mortality not attributable to the technique); e) Vayre (25) follows Ribault's technique and modifies the ileal suture by using a TEA and end-to-end anastomosis on a colon mucosa hammock, reinforcing it with Tissucol. There was no evidence of ileal colonization, and the histological study of the anastomosis showed a transition zone similar to an ileocecal valve; f) Blanco (4) carries out a valve by ileocolic intubation with a colon pouch. He observes a decrease in the number of stools; and g) Casal (5) follows Vayre technique using a GIA stapler instead of a TEA, with bilateral stapling of the ileal nipple. He reports increased pressure levels at the valve and absence of colonization.

In conclusion, when faced with the need for intestinal resection including the ileocecal valve, the surgical technique of choice would be our new valve, because of lower weight loss, lower number of stools, lower biochemical alterations, and absence of colonization of the ileal mucosa and bacterial retrograde contamination. The ileo-colic EE resection technique should be avoided whenever possible.

\section{REFERENCES}

1. Rombeau JL, Rolandelli RH. Enteral and intestinal adaptation in patients with enteric fistulas and short bowel syndrome. Surg Clin North Am 1987; 67: 551-71.

2. Canarelli JP. Réalisation expérimentale d'une valve iléo-colique conti-nen-te chez le rat. J Chir 1986; 123 (1): 39-44.

3. Ribault L, Veillard JM, Sarre B, Diouf B et al. Intubation iléo-colique droite après résection de l'iléon terminal pou péritonite géneralisée par perforation iléale. Expérience africaine de 33 cas. Chirurgie 1990; 116: 216- 28.

4. Blanco R. Construction of a new intestinal valve. Dis Colom Rectum 1994; 37 (6): 606-9.

5. Casal JE. Anastomosis ileocólica con intubación y pezón ileal. Colo Proctology 1996; vol XII, 96: 12-7 (ed español).

6. Tanner WA, O'Leary JF, Byrne PJ, Hennessy TPJ. The effect of reversed jejunal segments on the myoelectrical activity of the small bowel. Br J Surg 1978; 65: 567-71.

7. Périssat J. Résection massive de l'intestin grêle. Une technique d'anas-to-mose freinatrice du transit. J Chir 1974; 108: 179-86. 
8. Salomao J. Anastomose freinatrice du transit par rotation axiale de $180^{\circ}$ du segment intestinal d'amont après résection massive de l'intestin grêle. J Chir 1976; 112 (2): 99-6.

9. Mackby JM, Richard V, Gilfillans RS, Florida R. Methods of increasing the efficiency of residual small bowel segments. A preliminary study. Am J Surg 1965; 109: 32-8.

10. Budding J, Smich C. Role of recirculating loop in the management of massive resection of the small intestine. Surg Gynecol Obstet 1967; 125: 243-9.

11. de Vega DS. Reseccion intestinal masiva. ¿Son útiles los circuitos de re-circulación? Estudio experimental en perros. Rev Esp Enferm Dig 1986; 69 (3): 201-5

12. Layzell T, Collin J. Retrograde electrical pacing of small intestine: a new treatment for the short bowel syndrome? Br J Surg 1981; 68: 711-3.

13. de Lorimer AA, Harrison MR. Intestinal plication in the treatment of atresia. J Pediatr Surg 1983; 18: 734-7.

14. Weber TR, Vane DW, Grosfield JL. Tapering enteroplasty in infants with bowel atresia and short gut. Arch Surg 1982; 117: 684-8.

15. Schiller WR, DiDio LJA, Anderson MC. Production of artificial sphinc-ters. Ablation of the longitudinal layer of the intestine. Arch Surg 1967; 95: 436-42.

16. Blömer A, Dux A, Lenz H. Myotomie, eine chirurgische Methode zur
Verlangsammung der dürmdarm Passage. Biol Gastro-Enterol 1972; 5: 1972-8.

17. de Vega DS. Tratamiento quirúrgico del síndrome de intestino corto con una bolsa intestinal frenadora. Estudio experimental. Cir Esp 1984; 38 (2): 177-82.

18. Rodríguez Montes JA. Un intento de recirculación intestinal en perros con resección. Rev Quir Esp 1980; 57 (2): 149-54.

19. Binnington HB, Tumbleson ME, Ternberg JL. Use of jejunal neomucosa in the treatment of short gut syndrome in pigs. J Pediatr Surg 1975; 10: 617-21.

20. Wood RFM. Small bowel transplantation. Br J Surg 1992; 79: 193-4.

21. Bianchi A. Intestinal loop lengthening, a technique for increasing small intestinal length. J Pediatr Surg 1980; 15: 145-51.

22. Kimura K. A new bowel elongation technique for the short bowel syn-drome using the isolated bowel segment Iowa models. J Pediatr Surg 1993; 28 (6): 792-4.

23. Kellog JH. Technique of operation for repair of the ileo-caecal valve. Ann Surg 1913; 67-83.

24. Ricotta J, Zuidema GD, Gadacz TR, Dadri D. Construction of an ileocecal valve and its role in massive resection of the small intestine. Surg Gynecol Obstet 1981; 152: 310-4.

25. Vayre P. Rétablissement du circuit intestinal par trope iléal intracolique. Chirurgie 1991; 117: 318-28.

\title{
Nuevo tipo de neoválvula en la cirugía del intestino corto
}

\author{
M. Zurita, J. M. Raurich'1, A. Ramírez ${ }^{2}$ J. Gil² y J. Darder ${ }^{3}$ \\ Servicio de Cirugía General y Digestivo. ${ }^{1}$ Unidad de Cuidados Intensivos. ${ }^{2}$ Servicio de Microbiología. ${ }^{3}$ Servicio de \\ Anestesiología y Reanimación. Hospital Universitario Son Dureta. Palma de Mallorca, Islas Baleares
}

\section{RESUMEN}

El objetivo del estudio ha sido comparar la repercusión clínica y analítica de un nuevo tipo de válvula intestinal, aplicable tanto en las resecciones masivas intestinales como en las hemicolectomías derechas que requieren la eliminación de la válvula ileocecal, con dos series históricas: una con la técnica de resección ileocólica con anastomosis término-terminal (TT) y otra con la técnica válvular de Ricotta.

Se han comparado 23 pacientes en los que se aplicó la resección ileocólica TT, en 15 pacientes la formación de la válvula de Ricotta y en 20 pacientes a los que se aplicó la neoválula formada con la invaginación del intestino delgado en el colon. No hubo diferencias estadísticamente significativas en el peso inicial de los pacientes. Los pacientes con la neovávula presentaron una menor pérdida de peso y menor número de deposiciones a los 3 meses y al año comparados con las técnicas de Ricotta y especialmente con la resección ileocólica TT. Además con la neoválvula no hubo colonización de la mucosa ileal ni sobrecrecimiento bacteriano.

En conclusión ante las resecciones masivas intestinales y las hemicolectomías derechas que incluyan la válvula ileocecal, la técnica de elección sería la neoválvula.

Palabras clave: Síndrome de intestino corto. Válvula ileocecal. Sobrecrecimeinto bacteriano.

\section{INTRODUCCIÓN}

El síndrome de intestino corto (SIC) es el conjunto de síntomas y trastornos fisiopatológicos desencadenados tras una resección masiva intestinal (RMI) en la que puede incluirse total o parcialmente el colon, y siendo las causas más frecuentes el infarto mesentérico, la enfermedad de Crohn, la enteritis rádica, los traumatismos y los tumores (1). Las principales manifestaciones clínicas del SIC son la diarrea, la pérdida de peso y la desnutrición por malabsorción, especialmente cuando no es posible preservar el íleon terminal y la válvula ileocecal.

Distintas técnicas quirúrgicas se han aplicado para reducir las manifestaciones clínicas del SIC, siendo una de ellas la formación de válvulas. Con las técnicas quirúrgicas de la neoválvula se intenta conseguir desde el punto de vista funcional: a) disminuir la contaminación retrógrada bacteriana (CRB); b) disminuir el sobrecrecimiento bacteriano (SCB) y evitar una posible translocación bacteriana; c) disminuir la malabsorción y la diarrea; d) aumentar el tiempo de tránsito intestinal y el de absor- 
ción; e) disminuir la hiperoxaluria y la incidencia de litiasis renal y vesicular; f) reducir la pérdida de peso; y g) proporcionar una mejor calidad de vida al paciente. Aunque la experiencia clínica en la formación de neoválvulas en un SIC es limitada, se han descrito buenos resultados (2-5).

\section{OBJETIVOS DEL ESTUDIO}

Comparar la repercusión clínica y analítica de un nuevo tipo de válvula, aplicable tanto en la RMI como en las hemicolectomías derechas que requieran la eliminación de la válvula ileocecal, con dos series históricas una con la anastomosis ileocólica TT y otra con la técnica de la válvula de Ricotta.

\section{MATERIAL Y MÉTODOS}

\section{Descripción de la técnica de neoválvula por invaginación yeyunal y/o íleo cólica}

La aplicación de esta nueva técnica quirúrgica en patología humana fue realizada tras comprobaciones experimentales en perros adultos. Tras aplicar a los pacientes una laparotomía media se seleccionaban las zonas intestinales a resecar que eran seccionadas mediante un bisturí eléctrico. Posteriormente se procedía a colocar 3-4 puntos fiadores a $1 \mathrm{~cm}$ del borde del intestino delgado con sutura de doble aguja y colocados en sus bordes mesentérico, antimesentérico y laterales, los cuales son pasados de 1 en 1 a través de la luz del colon y exteriorizados en situación anatómica similar a la que le corresponden en el intestino delgado. La invaginación de intestino delgado en la luz cólica $(8 \mathrm{~cm})$ es facilitada por la mano y por una suave tracción de los 3-4 puntos fiadores y tras realizarla y comprobar que no existían rotaciones intestinales, se anudaban los puntos fiadores sobre la serosa cólica. Realizamos entonces circunferencialmente una corona de puntos sueltos con material irreabsorbible 3/0 entre el borde cólico seccionado y el intestino delgado, y una vez terminada, procedíamos a retirar los puntos fiadores lo que permitía de forma lenta pero progresiva la evaginación del intestino delgado invaginado y que prácticamente llegara hasta la línea de sutura colointestinal. Finalmente se procedía al cierre de la brecha mesocólica y se colocaba un drenaje de tipo penrose y cierre de la laparotomía (Fig. A, 1-5).

\section{Estudios clínicos}

Los pacientes fueron separados en 3 grupos según la técnica quirúrgica aplicada en los sucesivos periodos de tiempo: a) grupo ileocólica TT: intervención quirúrgica que consistía en una ileotransversostomía con anastomo- sis TT tras la resección intestinal, practicada entre los años 1975 y 1983; b) grupo Ricotta: aplicación de una válvula tipo Ricotta tras la resección intestinal, practicada entre los años 1984 y 1994; y c) grupo neoválvula: aplicación de la invaginación ileal en la luz cólica para formar una nueva válvula tras la resección intestinal, realizadas en el periodo 1995-2000.

Ileocólica TT. Se aplicó en 23 pacientes con una edad media de 68 años (64-73) y cuya relación varónhembra ha sido de 12:11. Se intervinieron 20 cánceres de colon derecho, 2 isquemias mesentéricas y 1 enfermedad de Crhon complicada.

Ricotta. Se utilizó en 15 pacientes con una edad media de 70 años (40-86), siendo la relación varón-hembra de 13:2. Se intervinieron 2 cánceres de colon derecho, 11 isquemias mesentéricas, 1 enfermedad de Crohn complicada y 1 linfoma ileal.

Neoválvula. Se realizó en 20 pacientes con una edad media de 70 años (59-83), siendo la relación varón-hembra de 12:8. Se practicó en 15 cánceres de colon derecho, 3 isquemias mesentéricas, 1 enfermedad de Crohn complicada y 1 linfoma ileal.

El seguimiento no fue posible en 3 pacientes (1 caso de anastomosis ileocólica T-T por isquemia mesentérica que dejó de acudir a las consultas; otro caso con la técnica de Ricotta por cáncer de ciego que rechazó por la edad cualquier tipo de exploración o estudio y un tercer caso al que se le había aplicado la construcción de la neoválvula por cambio de Comunidad Autónoma) y consistió en la práctica de un enema opaco, una colonoscopia y en un estudio de SCB mediante el test del aliento de hidrógeno con glucosa a partir de los 3, 6 y 12 meses de la intervención.

\section{Estadística}

Los resultados se expresan en su valor medio y la desviación estándar. Los estudios estadísticos fueron realizados aplicando la prueba de ANOVA.

\section{RESULTADOS}

Entre los 3 grupos de pacientes no hubo diferencias estadísticamente significativas en el peso inicial (Tabla I). A los 3 meses hubo una pérdida de peso que fue mínima en los pacientes del grupo a los que se aplicó la técnica de la Neoválvula y máxima en el grupo con la técnica ileocólica TT (Tabla I). Al año hubo una recuperación del peso que fue máxima con la técnica de la neoválvula y mínima con la técnica ileocólica TT (Tabla I).

El número de deposiciones post-cirugía a los 3 meses y al año fué menor con la técnica de la neoválvula y más elevada con la técnica ileocólica TT, siendo las diferencias estadísticamente significativas (Tabla I).

Los ácidos biliares a nivel sérico (rango normalidad: 0-8,1 $\mu \mathrm{mol} / \mathrm{l}$ ), ferritina (rango normalidad: 30-400 ng/dl) 
Tabla I. Comparación parámetros en patología clínica y según la técnica aplicada

\begin{tabular}{|c|c|c|c|c|}
\hline & Ileocólica T-T n: 23 & Ricotta $n: 15$ & Neoválvula $n: 20$ & $p$ \\
\hline Peso inicial & $67 \pm 6(59-80)$ & $67 \pm 7(58-81)$ & $70 \pm 4(62-78)$ & 0,10 \\
\hline Peso 3 meses & $51 \pm 5(42-66)$ & $57 \pm 6(50-69)$ & $64 \pm 4(57-73)$ & $<0,001$ \\
\hline Peso $\geq 1$ año & $58 \pm 5(52-68)$ & $63 \pm 7(51-76)$ & $68 \pm 4(61-76)$ & $<0,001$ \\
\hline Deposición postquirúrgica & $4 \pm 1(3-5)$ & $3 \pm 0,7(2-4)$ & $2,4 \pm 0,5(2-3)$ & $<0,001$ \\
\hline Deposición 3 meses & $3 \pm 0,3(2-3)$ & $2,3 \pm 0,4(2-3)$ & $1,8 \pm 0,4(1-3)$ & $<0,001$ \\
\hline Deposición $\geq 1$ año & $2 \pm 0,4(1-3)$ & $1,5 \pm 0,5(1-2)$ & $1,2 \pm 0,4(1-2)$ & $<0,001$ \\
\hline ABTS postquirúrgica $\mu \mathrm{mol} / \mathrm{l}$ & $1,2 \pm 0,6(0,2-2,3)$ & $1,5 \pm 0,5(1-2)$ & $2,5 \pm 0,9(0-4)$ & $<0,001$ \\
\hline ABTS 3 meses $\mu \mathrm{mol} / \mathrm{l}$ & $0,9 \pm 0,5(0-1,7)$ & $1,6 \pm 0,6(0,9-2,8)$ & $1,3 \pm 0,4(0,6-2)$ & $<0,001$ \\
\hline ABTS $\geq 1$ año $\mu \mathrm{mol} / /$ & $1,5 \pm 0,6(0-3)$ & $2,6 \pm 0,7(1-3,6)$ & $2 \pm 0,5(1-3)$ & $<0,001$ \\
\hline Ferritina postquirúrgica $\mathrm{ng} / \mathrm{ml}$ & $206 \pm 94(78-365)$ & $277 \pm 110(59-375)$ & $239 \pm 111(59-375)$ & 0,57 \\
\hline Ferritina 3 meses $\mathrm{ng} / \mathrm{ml}$ & $126 \pm 65(40-242)$ & $193 \pm 94(51-321)$ & $220 \pm 104(50-353)$ & 0,003 \\
\hline Ferritina $\geq 1$ año ng/dl & $183 \pm 81(70-310)$ & $203 \pm 98(56-337)$ & $233 \pm 109(55-377)$ & 0,25 \\
\hline Zinc postquirúrgica $\mu \mathrm{g} / \mathrm{dl}$ & $96 \pm 13(78-123)$ & $101 \pm 16(83-137)$ & $101 \pm 15(80-130)$ & 0,35 \\
\hline Zinc 3 meses $\mu \mathrm{g} / \mathrm{dl}$ & $65 \pm 8(52-79)$ & $87 \pm 13(71-116)$ & $92 \pm 18(62-124)$ & $<0,001$ \\
\hline Zinc $\geq 1$ año mg/dl & $81 \pm 10(69-100)$ & $92 \pm 14(74-122)$ & $103 \pm 14(79-127)$ & $<0,001$ \\
\hline Cobre postquirúrgica $\mu \mathrm{g} / \mathrm{dl}$ & $105 \pm 17(77-141)$ & $110 \pm 16(79-130)$ & $104 \pm 15(77-130)$ & $<0,001$ \\
\hline Cobre 3 meses $\mu \mathrm{g} / \mathrm{dl}$ & $152 \pm 35(100-243)$ & $126 \pm 17(91-150)$ & $112 \pm 16(83-140)$ & 0,51 \\
\hline Cobre $\geq 1$ año $\mu \mathrm{g} / \mathrm{dl}$ & $110 \pm 14(72-142)$ & $118 \pm 16(86-140)$ & $104 \pm 15(72-128)$ & $<0,001$ \\
\hline
\end{tabular}

ABTS: ácidos biliares totales nivel sérico

y zinc (rango normalidad: $80-130 \mu \mathrm{g} / \mathrm{dl}$ ) presentaron una caída a los 3 meses con tendencia a la normalización al año, siendo la caída más acusada con la técnica ileocólica TT. El cobre (rango de normalidad: $70-140 \mu \mathrm{g} / \mathrm{dl}$ ) presentaba un incremento en sus valores de bases más acusado con la técnica ileocólica TT (Tabla I).

Comparando los pacientes en los que se practicó una hemicolectomía derecha ampliada con los sometidos a una RMI, el número de deposiciones fue menor con las técnicas de la neoválvula y de Ricotta que con la técnica ileocólica TT y este menor ritmo deposicional se mantiene en el tiempo (Tabla II). Entre la neoválvula descrita y la técnica de Ricotta las diferencias no alcanzaron significado estadístico (Tabla II).

Los enemas opacos practicados a 17 pacientes con anastomosis ileocólica TT evidenciaron un relleno retrógrado importante en el intestino delgado. Los 15 pacientes del grupo neovalvular y los 10 del grupo tipo Ricotta mostraron una válvula continente, sin obstrucción ni reflujo en los enemas opacos realizados (Fig. A, 6).

Las colonoscopias practicadas en 15 pacientes del grupo de la neoválvula, en 8 del grupo Ricotta y en 9 del grupo ileocólica TT evidenciaron: todos los pacientes con anastomosis ileocóloca TT evidenciaron colonización de la mucosa ileal; colonización de menor intensidad en 4 pacientes sometidos a la técnica de Ricotta y ausencia de colonización ileal con la técnica de la neoválvula (Figs. B, 1-5 y B, 6).

Los resultados de los estudios de SCB practicados mediante el test del aliento de hidrógeno con glucosa, fueron: con la técnica ileocólica TT positivos en todos los pacientes a los 3 meses y en 4 casos al año; con la técnica de Ricotta fueron positivos para SCB en 2 pacientes a los 3 meses y en ningún paciente al año y con la técnica de la neoválvula todos los pacientes fueron negativos para el SCB a los 3 y 12 meses.

\section{DISCUSIÓN}

Los principales resultados del estudio comparativo de las tres técnicas quirúrgicas, indican que con la técnica de la neoválvula los pacientes presentan menor pérdida de peso, menor frecuencia de deposiciones y menor alteración bioquímica, todo lo contrario que con la técnica ileocólica TT. Así mismo, se evidencia la ausencia de colonización de la mucosa ileal y de contaminación retrógrada bacteriana con las técnicas de las neoválvulas en comparación con la técnica ileocólica TT.

Las distintas técnicas quirúrgicas que se han aplicado para reducir las manifestaciones clínicas del SIC son: a) asas intestinales antiperistálticas (6-8); b) asas intestinales de recirculación (9-11); c) marcapasos eléctrico retrógrado (12); d) reducción del calibre y plicatura intestinal $(13,14)$; e) miotomías intestinales $(15,16)$; f) bolsas intestinales (17); g) anillos periintestinales (18); h) crecimiento de neomucosa intestinal (19); i) transplante de intestino delgado $(20) ; \mathrm{j}$ ) reducción del calibre y alargamiento del intestino delgado (21); k) alargamiento secuencial (22); y l) formación de válvulas.

Respecto a la formación de válvulas, en animales de laboratorio sometidos a un SIC, son varios los tipos utilizados y observándose una mayor supervivencia. El primer estudio bibliográfico encontrado es el de Kellog (24) y basados en estos trabajos, Ricotta (25) en 1981 y tras la realización de urgencia en un niño de una RMI, practica una intubación yeyuno-cólica tipo pezón de $8 \mathrm{~cm}$ de longitud y en 2 planos, haciendo posteriormente un estudio experimental en perros con válvula, observando el efecto frenador de la misma. Tras las constataciones experimentales y clínicas podemos indicar que el restablecimiento del tránsito intestinal es simple, sin apenas morbi-mortalidad y que puede ser comparada esta invaginación a la 
Tabla II. Comparación clínica entre la hemicolectomía D ampliada y las RMI-TT según la técnica quirúrgica aplicada: la neoválvula (NV), la resección ileocólica término-terminal (RMI-TT) y la técnica de Ricotta (Ricotta).

\begin{tabular}{|c|c|c|c|c|c|c|}
\hline & \multicolumn{3}{|c|}{ Hemicolectomía D } & \multicolumn{3}{|c|}{$R M I$} \\
\hline & $N V$ & $R M I-T T$ & Ricotta & $N V$ & RMI-TT & Ricotta \\
\hline $\mathrm{N}^{\circ}$ pacientes & 13 & 20 & 2 & 7 & 3 & 13 \\
\hline $\mathrm{N}^{\circ}$ deposiciones $<3$ meses & $2,5^{*}$ & 3,5 & 2,6 & $3 *$ & 6 & 3,5 \\
\hline $\mathrm{N}^{\circ}$ deposiciones $>3$ meses & $1,3 *$ & 2,5 & $1,5^{*}$ & $2 *$ & 5,5 & $2,2 *$ \\
\hline No deposiciones (1-5 años) & 1 * & 2,2 & $1 *$ & $1,2^{*}$ & 3,6 & $1,7 *$ \\
\hline Fuga anastomótica & 1 & 2 & 0 & 0 & 0 & 2 \\
\hline Exitus por intervención & 0 & 0 & 0 & 0 & 0 & 0 \\
\hline Exitus por otra causa & 1 & 3 & 0 & $2++$ & 1 & 2 \\
\hline
\end{tabular}

*p $<0,05$ respecto a RMI-TT

++pacientes en fallo multiorgánico preestablecido

Tabla III. Parámetros comparativos en patología clínica y técnicas

\begin{tabular}{|c|c|c|c|c|c|c|}
\hline & Ricotta & Ribault & Vayre & Blanco & Casal & Autores \\
\hline$\overline{\text { Año }}$ & 1981 & 1990 & 1991 & 1994 & 1996 & 2000 \\
\hline Flora luz de ID-IG & No & No & No & No & No & Sí \\
\hline Flora propia mucosas intestinales & No & No & No & No & No & Sí \\
\hline Estudio RX baritado & Sí & No & Sí & Sí & Sí & Sí \\
\hline Tiempo tránsito intestinal & Sí & $\mathrm{Ne}$ & $\mathrm{Ne}$ & Sí & $\mathrm{Ne}$ & Sí \\
\hline $\mathrm{N}^{\circ}$ deposiciones/día & Sí & No & Sí & Sí & No & Sí \\
\hline Eco vesicular y renal & No & No & No & No & No & Sí \\
\hline Obstrucción/fístula & Sí & Sí & No & No & No & No \\
\hline Colonización retrógrada & Sí & $\mathrm{Ne}$ & $\mathrm{Ne}$ & $\mathrm{Ne}$ & No & No \\
\hline SCB: prueba aire espirado & No & No & No & No & No & Sí \\
\hline Síndrome estenótico & Sí & Sí & No & $\mathrm{Ne}$ & No & No \\
\hline Mortalidad postoperatoria & $\mathrm{Ne}$ & $15 \%$ & $18 \%$ & No & No & $15 \%$ \\
\hline
\end{tabular}

ID: intestino delgado; IG: intestino grueso; Ne: no especificado; SCB: sobrecrecimiento bacteriano.

válvula ileocecal. Al evaginarse la mucosa ileal se pone en contacto con la mucosa cólica hasta el nivel de la línea de sutura externa colo-ileal, evitando dicha evaginación el riesgo de estenosis por la coalescencia de los bordes de la invaginación ileal; la mucosa ileal es la que está en contacto con el contenido intracólico, lo que es preferible a que lo sea la superficie serosa. Esta evaginación ileal permite el restablecimiento del tránsito tras una RMI, siendo pues útil en el SIC. Creemos que la neoválvula es continente y permite alcanzar todos los objetivos del estudio.

Revisión histórica de la formación de algunos tipos de neoválvulas (Tabla III): a) Kellog (23) intenta reparar la válvula ileocecal incompetente mediante la invaginación del íleon terminal en el ciego; b) Ricotta (24) realiza una neoválvula ileocólica en un SIC en un niño y tras estudios experimentales y en aplicaciones clínicas encontró menor pérdida de peso y presencia de SCB en el ID residual; c) Canarelli (2) forma una hemivalva siguiendo la técnica de Ricotta. Reduce el tiempo de tránsito sin encontrar oclusiones; d) Ribault (3) realiza una intubación ileocólica tipo Ricotta sobre una hamaca mucosa de colon en enfermedades tifoideas intestinales complicadas.
Informa de escasas complicaciones (oclusión y fístula un $3 \%$ y el $15 \%$ de mortalidad no atribuible a la técnica); e) Vayre (25) sigue la técnica de Ribault modificando la sutura ileal mediante el uso de una TEA y con anastomosis término-terminal sobre hamaca mucosa en el colon reforzando la misma con tissucol. No evidencia colonización ileal y en el estudio histológico de la anastomosis encuentra una zona de transición similar a la válvula ileocecal; f) Blanco (4) realiza una válvula por intubación ileocólica con un bolsillo cólico. Observa disminución del número de deposiciones; y g) Casal (5) sigue la técnica de Vayre utilizando una GIA en lugar de TEA con grapado bilateral del pezón ileal. Informa de un incremento de los niveles de presión de la válvula y ausencia de colonización.

En conclusión, ante la necesidad de una resección intestinal que incluya la válvula ileocecal, la técnica quirúrgica de elección debería ser la neoválvula por la menor pérdida de peso, el menor número de deposiciones, la menor alteración bioquímica y la ausencia de colonización de la mucosa ileal y de contaminación retrógrada bacteriana. La técnica quirúrgica de resección ileocólica TT debería evitarse siempre que fuese posible. 